\title{
The Role of Synovial Fluid Aspiration in Shoulder Joint Infections
}

\author{
Laura Elisa Streck \\ Koenig-Ludwig-Haus Wuerzburg, University of Wuerzburg \\ Johannes Forster \\ Insititute for Microbiology and Hygiene, University of Wuerzburg \\ Sebastian Philipp von Hertzberg-Boelch \\ Koenig-Ludwig-Haus Wuerzburg, University of Wuerzburg \\ Thomas Reichel \\ Koenig-Ludwig-Haus Wuerzburg, University of Wuerzburg \\ Maximilian Rudert \\ Koenig-Ludwig-Haus Wuerzburg, University of Wuerzburg \\ Kilian Rueckl ( $\nabla$ kilian.rueckl@gmail.com ) \\ Koenig-Ludwig-Haus Wuerzburg, University of Wuerzburg
}

\section{Research Article}

Keywords: shoulder joint infection, periprosthetic joint infection, synovial fluid white blood cell count, joint aspiration, microbiological culture, interstage aspiration

Posted Date: August 5th, 2021

DOI: https://doi.org/10.21203/rs.3.rs-721939/v1

License: () (1) This work is licensed under a Creative Commons Attribution 4.0 International License. Read Full License 


\section{Abstract}

Background: Joint aspiration with analysis of synovial fluid white blood cell count (WBC) and microbiological culture is a widely established aspect in the diagnosis of shoulder joint infections (SJI). In case of a two stage revision for SJI, joint aspiration before re-/implantation of an total shoulder arthroplasty (TSA) was used to rule out persistent infection for years but its value is under debate. Shoulder specific data on all aspects is rare. The current study aims to answer the following research questions: Joint aspiration has an insufficient predictive value in the diagnosis of SJI in (1) initial workup and (2) before definite arthroplasty with polymethylmethacrylate (PMMA)-Spacer in place.

Methods: This retrospective evaluation investigates 35 patients that were treated for SJI with a two staged implantation of a TSA after debridement and implantation of an PMMA-Spacer. Joint aspirations were performed preoperatively (PA) and before re-/implantation of the prosthesis while spacer was in place (interstage aspiration, IA). Samples were taken for microbiological culture and analysis of WBC. Sensitivity and specificity were calculated with reference to intraoperative microbiological samples. Receiver Operating Characteristic (ROC), Area-Under-Curve analysis (AUC) and calculation of the Youden index were performed to find optimum cut-off for WBC.

Results: The sensitivity of microbiological cultures from PA was $58.3 \%$ and the specificity was $88.9 \%$. The mean WBC was 27800 leucocytes/mm ${ }^{3}$ (range $400-96300$ ). The maximum Youden index (0.857) was a cut-off of 2600 leucocytes $/ \mathrm{mm}^{3}$ with a sensitivity of $85.7 \%$ and a specificity of $100.0 \%$. The sensitivity and specificity of IA were $0.0 \%$ and $88.5 \%$, respectively.

Conclusions: PA is likely to miss Cutibacteria spp. Cuand CoNS and cannot rule out infection for sure. However, we recommend PA for its advantages of targeted antibiotic therapy in case of germ identification. Empiric antibiotic therapy should therefore cover these bacteria even if aspiration showed negative microbiological cultures. In contrast, diagnostic value of IA does not qualify for routine use.

Trial registration: The study was approved by the ethic committee of the author`s institution (registration number 235/16-mk). The patients gave written informed consent to their inclusion in the study.

\section{Background}

Joint infections are a serious condition both after surgical intervention as well as in the native joint. It often results in multiple revision surgeries, relevant loss of function in the affected extremity and is accompanied by a high mortality [4-7]. The early and reliable diagnosis of an infection is essential for effective treatment and prevention of generalized, septic progressions (1-6). Synovial fluid aspiration is a central pillar in the diagnostic workup. Diagnostic markers from synovial fluid include white blood cell count (WBC) with polymorphonuclear percentage, leukocyte esterase level, alpha-defensin level, synovial CRP and microbiological culture. WBC has been used as a reliable tool in the diagnosis of infections of the lower extremity but shoulder specific data is limited to scattered and small studies (7). To date, there is no consensus about cut-off values. The direct transfer of values from the lower extremity does not seem to be adequate (8). The specific bacterial spectrum with Cutibacterium spp. and coagulase-negative staphylococci (CoNS) further complicates the assessment of results of microbiological cultures and increases the risk of false negative results $(3,9,10)$. The reported sensitivity of PA widely varies between $9-85 \%$ and its value is under debate $(11,12,9,10,13)$.

This uncertainty applies even more for joint aspiration with PMMA-spacer in place during two-stage revision surgery, a common therapy regimen for periprosthetic shoulder infections(14-16). It was common practice to perform a joint aspiration after 14 days of antibiotic suspension and await the microbiologic culture before final TSA implantation. This procedure has widely been abandoned in two-stage revision for periprosthetic infections of the hip and knee as sensitivity and specificity of this "interstage aspiration" were reported low[19-21]. Shoulder specific data is largely absent. However, it is doubtful whether a transfer of the experience gained in hip and knee surgery to shoulder infections is justified. Therefore, it is crucial to gather joint specific data on SJI to improve diagnostic tools, scores and workflows.

The current study aims to answer the following research questions: Does joint aspiration has an insufficient predictive value in diagnose of shoulder joint infections (SJI) in (1) initial workup and (2) before definite arthroplasty with PMMA-Spacer in place?

\section{Materials And Methods}

This retrospective evaluation investigated consecutive patients that were treated for SJI with a two staged implantation of a total shoulder arthroplasty (TSA) after debridement and implantation of an antibiotic loaded PMMA-Spacer between 2007 and 2015 in one specialized high volume hospital. Inclusion criteria were 1) the diagnosis of SJI based on clinical presentation, blood infection markers, histological and microbiological findings as well as WBC following $2018 \mathrm{~s}$ ICM criteria (17), 2) the two staged TSA implantation as described above and 3) available data on PA and IA. Exclusion criteria was age below 18, this criteria was not met in a patient who fitted the inclusion criteria. We identified 35 patients that were eligible for the study (23 periprosthetic shoulder infections (PSI), 8 native joint infections (primary infection, PI), 7 infections after osteosynthesis and/or rotator cuff surgery (secondary infection, SI). The first joint aspiration was performed preoperatively (PA); the second aspiration was performed as interstage aspiration while a spacer was implanted (IA). Patients did not receive antibiotics before PA, all patients were treated with antibiotics after spacer implantation but an antibiotic suspension of 14 days was held before performing IA.

Data for certain aspects was not available in all of the patients, therefore sample sizes differ for different questions. Culture from PA: 35 patients (7 PI, 21 PSI, $8 \mathrm{SI}$ ), WBC: 12 patients (3 PI, 9 PSI), IA: 33 patients (6 PI, $21 \mathrm{PSI}, 7 \mathrm{SI}$ ). Details on demographic data in blood infection markers are provided in Table 1.

Joint aspirations were performed with a sterile canula from either anterior or dorsal approach after 3 minutes skin disinfection by alcoholic skin disinfectant Loading [MathJax]/jax/output/CommonHTML/jax.js dt, Germany). A minimum volume of $1 \mathrm{ml}$ was required. Aspirated fluid volume was never diluted by using other 
fluids. Aspirated synovial fluid was transferred to a sterile test tube forWBC calculation and to blood culture bottles for aerobic (BacT/ALERT ${ }^{\circledR}$ FA Plus) and anaerobic (BacT/ALERT® FN Plus) growth (bioMérieux, Marvie-L

$\mathrm{E} \rightarrow i \leq$, France $)$. Bloodcturevialswere $\in$ cubatedf or 14 days or untilflag $\geq$ dpositive $\in$ theBac $\frac{T}{A} L E R T ® 3 D S y s t e m($ bioMérieux, Marvie $-I$ Étoile, France). Species diagnosis was established by Matrix-assisted laser desorption/ionization - time of flight (MALDI-TOF) from solid culture media.

Statistical tests were performed using SPSS Statistics ${ }^{\circledR}$ Version 24.0 (IBM, New York, USA) and Microsoft Excel® Version 1908 (Microsoft, Washington, USA). Descriptive statistics were performed to describe means, medians and range for all variables. Receiver Operating Characteristic (ROC) was plotted for WBC depending on culture results from spacer implantation to depict the correlation between sensitivity and specificity. Area-Under-Curve (AUC) analysis and calculation of the Youden index was performed to quantify the quality of the test. The same calculations were performed for WBC depending on culture results from PA.

Microbiological culture from PA was defined as "correct positive" if a microbiological culture from tissue biopsies taken during initial surgery matched the organism of PA. PA was defined "correct negative" if neither the culture from PA nor intraoperative biopsies yielded growth. PA was defined "false negative" if the culture from PA was negative while an intraoperative biopsy yielded growth. PA was defined "false positive" if culture from PA yielded growth and intraoperative biopsies yielded growth of a different bacterium (1 patient) or no growth.

The intraoperative biopsies were taken right after opening the joint capsule and before starting perioperative intravenous antibiotics. At least 3 biopsies were taken from membranes, resected bone and directly surrounding soft tissue. Sterile sample vessels were opened under laminar flow and fractioned. Cultures were nourished in Brain-Heart-Infusion-Bouillon and Thyoglycolate-Bouillon and plated out on Candida-Chrome-Agar, Columbia-Blood-Agar, Cooking-BloodAgar, MacConkey-Agar and Schaedler-Agar. Cultures were incubated for 14 days. Species were differentiated throughout mass spectrometry (Vitek MS, bioMérieux, Marvie-LÉ $\rightarrow i \leq$, France)followedbytest $\in$ gofsensitivity(Vitek2, bioMérieux, Marvie-LÉtoile, France).

Sensitivity was defined as the ratio of all correct positive PAs on all intraoperative positive cultures. Specificity was defined as the ratio of all positive PAs on all intraoperative positive cultures. The same procedure was used for IAs but with reference to results from either spacer exchange or final implantation of TSA. Cross-tables were used to calculate positive predictive value (PPV) and negative predictive value (NPV) for both PA and IA. 
Table 1

Demographic data and preoperative blood infection markers. CRP = C-reactive protein (norm value $<0,8 \mathrm{mg} / \mathrm{dl}$ ), $\mathrm{ESR}=\mathrm{erythrocyte}$ sedimentation rate (norm value $<28 \mathrm{~mm} / \mathrm{h}$ ). * IA was delayed due to intermediate cardic surgery/treatment for pulmonary problems. This two patients were excluded for the calculation of means (marked with **).

\begin{tabular}{|c|c|c|c|c|c|c|c|c|c|}
\hline patient & sex category & site & initial surgery & age at PA & surgery to PA & spacer to IA & CRP & ESR & blood WBC \\
\hline & & & & [years] & [months] & [days] & [mg/dl] & {$[\mathrm{mm} / \mathrm{h}]$} & {$\left[10^{3} / \mathrm{mm}^{3}\right]$} \\
\hline 1 & female & right & osteosynthesis & 44 & 18 & 55 & 1.6 & 34 & 7.6 \\
\hline 2 & female & right & no surgery & 74 & & 48 & 4.7 & 67 & 6.1 \\
\hline 3 & female & left & no surgery & 71 & & 32 & 2.5 & & 10.5 \\
\hline 4 & malefte & right & TSA & 55 & 35 & 34 & 8.5 & 23 & 9.5 \\
\hline 5 & female & right & TSA & 71 & 14 & 15 & 4 & & 9.5 \\
\hline 6 & female & right & TSA & 80 & 32 & 40 & 6.8 & 82 & 8.8 \\
\hline 7 & female & left & osteosynthesis & 68 & 4 & 52 & 2.9 & 64 & 10.7 \\
\hline 8 & female & right & TSA & 57 & 11 & 58 & 2.4 & 31 & 12.2 \\
\hline 9 & male & left & osteosynthesis & 52 & 8 & 52 & 1.5 & & 7.1 \\
\hline 10 & female & right & TSA & 84 & 4 & 57 & & 21 & \\
\hline 11 & male & right & TSA & 59 & 155 & 38 & 1.2 & & 7.8 \\
\hline 12 & male & right & rotator cuff & 68 & 32 & 44 & 1.3 & 18 & 8.6 \\
\hline 13 & female & right & TSA & 77 & 56 & $318^{*}$ & 0.4 & & 6.6 \\
\hline 14 & female & right & osteosynthesis & 66 & 2 & 50 & 1.1 & & 5.4 \\
\hline 15 & female & left & TSA & 74 & 16 & 46 & 0.4 & & 9.4 \\
\hline 16 & male & left & TSA & 74 & 13 & 58 & 1.7 & 18 & 11.4 \\
\hline 17 & male & right & TSA & 55 & 10 & 41 & 2.6 & 64 & 8.1 \\
\hline 18 & male & right & TSA & 73 & 24 & 44 & 11.3 & 32 & 8.3 \\
\hline 19 & male & right & TSA & 84 & 8 & 86 & 1.5 & 40 & 4.4 \\
\hline 20 & female & right & TSA & 65 & 14 & 50 & 4.8 & & 8.8 \\
\hline 21 & male & right & TSA & 72 & 1 & 74 & 30.4 & 68 & 8.6 \\
\hline 22 & male & right & TSA & 76 & 2 & 48 & 10 & 44 & 11.1 \\
\hline 23 & female & right & TSA & 60 & 9 & 44 & 1.4 & & 6.6 \\
\hline 24 & female & left & TSA & 78 & 37 & 56 & 12.1 & 40 & 13.8 \\
\hline 25 & female & right & no surgery & 76 & & $332^{*}$ & 1.6 & & 7.2 \\
\hline 26 & female & right & TSA & 77 & 28 & 56 & 0.1 & 38 & 5.6 \\
\hline 27 & female & right & no surgery & 82 & & 41 & 1.9 & 29 & 7.6 \\
\hline 28 & female & right & no surgery & 84 & & 20 & 7.1 & 8 & 10.3 \\
\hline 29 & female & right & TSA & 69 & 101 & 58 & 0.1 & 24 & 7.5 \\
\hline 30 & female & left & no surgery & 83 & & 59 & 0.3 & & 8.3 \\
\hline 31 & female & right & no surgery & 79 & & & 6.6 & 64 & 6 \\
\hline 32 & female & left & osteosynthesis & 65 & 2 & 59 & 1 & 31 & 10.5 \\
\hline 33 & female & left & TSA & 84 & 69 & 56 & 1.8 & 6 & 11.7 \\
\hline 34 & male & left & rotator cuff & 50 & 127 & & 0 & 29 & 5.4 \\
\hline 35 & female & right & TSA & 80 & 10 & 58 & 9.7 & 1 & 21.8 \\
\hline \multicolumn{4}{|c|}{ mean (only patients with TSA) } & 72 & 31 & $47^{\star \star}$ & 5.5 & 35 & 9.5 \\
\hline \multicolumn{4}{|c|}{ mean (patients with non TSA) } & 69 & 28 & 51 ** & 2.4 & 38 & 7.9 \\
\hline \multicolumn{4}{|c|}{ mean (total) } & 70 & 30 & $49^{* *}$ & 4.3 & 37 & 8.9 \\
\hline
\end{tabular}




\section{Results}

\section{Microbiological Culture from Preoperative Aspiration}

PA did not provide required minimum fluid volume in $11 / 35$ cases. The remaining 24 cases had positive cultures in $42 \%$ of cases. Within this positive cultures, bacteria detected was 30\% Cutibacterium spp., 20\% Corynebacterium spp. and 50\% others (Enterococcus faecalis, Escherichia coli, Staphylococcus aureus, Streptococcus viridans in $10 \%$ respectively and further $10 \%$ bacteria who yieldedobligate anaerobe growth in blood culture bottles but species diagnose was impeded by insufficient growth on solid media). Figure 1 depicts results of microbiological cultures from PA. Sensitivity was $58.3 \%$, specificity was $88.9 \%$. PPV was 0.88 , NPV was 0.64 . Table 2 depicts detailed information on microbiological results.

\section{Synovial Fluid White Blood Cell Count}

Mean WBC was 27800 leucocytes/mm $\mathrm{mm}^{3}$ (range 400-96300). Results of WBC analysis are presented in Fig. 2. Compared to microbiologic culture from spacer implantation, the maximum Youden index (0.886) was a cut-off of 2600 leucocytes $/ \mathrm{mm}^{3}$ with a sensitivity of $85.7 \%$ and a specificity of $100.0 \%$ ( $\left.p<0,001\right)$. ROC-AUC results are presented in Fig. 3. Mean WBC in patients with periprosthetic infections was 37300 leucocytes/mm ${ }^{3}$ (range 400-96300). The maximum Youden index $(0,893)$ was a cut-off of 700 leucocytes $/ \mathrm{mm}^{3}$ with a sensitivity of $85.7 \%$ and a specificity of $100.0 \%$ ( $\left.p<0,001\right)$. ROC-AUC results are presented in Fig. 4. Table 2 depicts detailed information on WBC.

\section{Interstage Aspiration}

IA did not provide required minimum fluid volume in $3 / 33$ cases. The remaining 30 cases showed positive cultures in $7 \%$. Figure 5 depicts results of microbiological cultures from IA. Bacteria detected were Staphylococcus epidermidis, Paenibacillus spp. and Paracoccus spp. (one each). The sensitivity was $0.0 \%$ and the specificity was $88.5 \%$ with a PPV of 0.00 and a NPV of 0.92 . Table 2 depicts detailed information on the microbiological results. 
Table 2

WBC and results of microbiological cultures in patients with shoulder joint infections.

\begin{tabular}{|c|c|c|c|c|c|c|}
\hline \multicolumn{7}{|c|}{ SYNOVIAL WBC AND MIRCROBIOLOIG CULTURE RESULTS } \\
\hline patient & initial surgery & $\begin{array}{l}\text { WBC from PA } \\
\left.\text { [cells } / \mathrm{mm}^{3}\right]\end{array}$ & $\begin{array}{l}\text { culture from } \\
\text { PA }\end{array}$ & $\begin{array}{l}\text { culture from spacer } \\
\text { implantation }\end{array}$ & $\begin{array}{l}\text { culture from } \\
\text { IA }\end{array}$ & $\begin{array}{l}\text { culture from spacer exchange/TSA } \\
\text { implantation }\end{array}$ \\
\hline 1 & osteosynthesis & & insufficient & CoNS & negative & \\
\hline 2 & no surgery & & insufficient & S. epidermidis & negative & negative \\
\hline 3 & no surgery & & negative & & negative & negative \\
\hline 4 & TSA & 400 & negative & Cutibacteria & negative & negative \\
\hline 5 & TSA & & insufficient & S. epidermidis & negative & negative \\
\hline 6 & TSA & & E. faecalis & & insufficient & negative \\
\hline 7 & osteosynthesis & & $\begin{array}{l}\text { Corynebacteria } \\
\text { spp. }\end{array}$ & & negative & negative \\
\hline 8 & TSA & & $\begin{array}{l}\text { anaerobic } \\
\text { bacteria }\end{array}$ & F. magna & $\begin{array}{l}\text { Paracoccus } \\
\text { spp. }\end{array}$ & negative \\
\hline 9 & osteosynthesis & & insufficient & S. epidermidis & negative & negative \\
\hline 10 & TSA & & negative & S. aureus & negative & negative \\
\hline 11 & TSA & & insufficient & negative & negative & \\
\hline 12 & rotator cuff & & negative & negative & negative & negative \\
\hline 13 & TSA & & insufficient & Cutibacteria & negative & negative \\
\hline 14 & osteosynthesis & & insufficient & S. epidermidis + C. acnes & negative & negative \\
\hline 15 & TSA & 400 & E. coli & negative & negative & negative \\
\hline 16 & TSA & 23400 & negative & S. epidermidis & negative & negative \\
\hline 17 & TSA & & negative & negative & negative & C. acnes \\
\hline 18 & TSA & & negative & C. acnes & $\begin{array}{l}\text { Paenibacillus } \\
\text { spp. }\end{array}$ & negative \\
\hline 19 & TSA & & insufficient & C. acnes & negative & S. warneri \\
\hline 20 & TSA & 56200 & $\begin{array}{l}\text { Corynebacteria } \\
\text { spp. }\end{array}$ & C. avidum & $\begin{array}{l}\text { S. } \\
\text { epidermidis }\end{array}$ & negative \\
\hline 21 & TSA & & insufficient & gramnegative strains & negative & negative \\
\hline 22 & TSA & 76800 & C. acnes & C. acnes & negative & negative \\
\hline 23 & TSA & 36600 & S. aureus & S. aureus & negative & negative \\
\hline 24 & TSA & 96300 & S. viridans & S. viridans & negative & negative \\
\hline 25 & no surgery & & negative & negative & negative & negative \\
\hline 26 & TSA & & negative & S. xylosus & negative & negative \\
\hline 27 & no surgery & & insufficient & negative & negative & negative \\
\hline 28 & no surgery & 1200 & negative & negative & negative & negative \\
\hline 29 & TSA & 700 & negative & negative & insufficient & S. epidermidis \\
\hline 30 & no surgery & 2600 & negative & negative & negative & negative \\
\hline 31 & no surgery & 400 & negative & negative & & negative \\
\hline 32 & osteosynthesis & & insufficient & S. epidermidis & negative & negative \\
\hline 33 & TSA & 45000 & C. avidum & C. avidum & negative & negative \\
\hline 34 & rotator cuff & & C. acnes & C. acnes & & negative \\
\hline 35 & TSA & & negative & negative & insufficient & S. epidermidis \\
\hline
\end{tabular}

\section{Discussion}


PA is a widely established method for the diagnosis of shoulder infection but its value is under debate. This debate is not at least caused by a sensitivity of PA reported within a wide range between $9-85 \%(11,12,9,18)$. The current study revealed a sensitivity of $58 \%$ for microbiological culture from PA. This is inferior to the reported sensitivity of intraoperative samples (14). In particular, the detection rate of Cutibacterium spp. was low and CoNS were not detected at all. This is remarkable especially against the background that Cutibacterium spp. and CoNS are described as the main pathogens causing shoulder joint infections (14, $12,19,20,18)$. Their detection by aspiration is hampered as both are slow growing organisms and both are able to produce biofilms. Therefore, even while being present as sessile pathogens within a biofilm in a tissue, they might not be detectable in an aspirate in a planktonic state (21). Second, Cutibacterium spp. are anaerobe bacteria and they are prone to missed by culture due to inadequate preanalytic process such as suboptimal culture media or excessive long transport time (22). Another controversially discussed aspect is the adequate period of incubation. Pottinger et al. described that only $86 \%$ of positive Cutibacterium acnes cultures were positive within 14 days (23). In contrary, Frangiamore et al. considered true positive cultures to be positive within 4-6 days(24). The incubation period of 14 days, used for the current study, is in accordance to other authors (8). Notwithstanding this discussion, the high rate of false negatives (42\%) of the current study stands in line with previous reports $(2,6,7,25)$ and disqualifies microbiological cultures from PA to rule out infection. However, the herein high specificity of PA of $89 \%$ endorse PA as an important diagnostic pillar.

Alongside cultures, PA provides WBC. There is no consensus relating to a cut-off value for infection suspicion (26, 27). To our best knowledge, very few studies exist that offer shoulder specific data. Nodzo et al. reported WBC in patients with periprosthetic $C$. acnes infections of the shoulder, hip and knee. Mean WBC for the PSI was 750 leucocytes/ $\mathrm{mm}^{3}$. This was closest to values for the hip (500 leucocytes $\left./ \mathrm{mm}^{3}\right)$ (8). Based on this study, 2018s International Consensus Meeting on periprosthetic joint infection in Philadelphia (ICM) suggested that PSI seems to be most likely comparable to low-grade periprosthetic hip infections and therefore recommended a cut-off of 3000 leucocytes $/ \mathrm{mm}^{3}$ (28). Strahm et al. who calculated WBC in 19 periprosthetic shoulder infections and suggested a threshold of 12200 leucocytes $/ \mathrm{mm}^{3}$ (29). The mean WBC of 27800 leukocytes $/ \mathrm{mm}^{3}$ is clearly suspicious for infection. However, there was a wide range of WBC (400-96300 leucocytes/ $\mathrm{mm}^{3}$ ). Based on our data, a cut-off value of 2600 leucocytes $/ \mathrm{mm}^{3}$ revealed a sensitivity of $86 \%$ and specificity of $100 \%$ and therefore provides an excellent validation for PSI. For the subgroup of periprosthetic infections, a maximum Youden index was reached at a threshold of 700 leucocytes $/ \mathrm{mm}^{3}$. While this result is based on a limited number of patients, it calls into question that periprosthetic infections of the shoulder come along with higher WBC than native or implant associated infections of the shoulder. Regardless of this point of discussion, our results implicate that WBC amongst various diagnostic parameters provided the best sensitivity to detect infection, yet a negative WBC cannot be used to rule out infection. We suggest that the direct adaption of values from hip infections to the shoulder might not be adequate. As our sample size was limited, further studies with enhanced sample size should follow to validate the current shoulder specific data on this important diagnostic parameter.

IA showed a devastating sensitivity of $0 \%$. The problem of minimal detection rates is also known for periprosthetic infections of the hip and the knee. Boelch et al. described a sensitivity of $5 \%$ for the hip respectively $0 \%$ for the knee $(30,31)$. In parallel, a shift from negative aspirations to low-virulent, biofilm-forming bacteria in intraoperative cultures is described (31). It seems likely that this effect is even more relevant in shoulder infections as Cutibacterium spp. and CoNS are typical slow-growing, low-virulent organisms (32). Our data supports these considerations to that effect, that most false negative samples showed cutibacterium acnes in intraoperative cultures and the two cases with intraoperative detection of staphylococcus epidermidis had resulted in dry taps before. Our study revealed a good specificity of $89 \%$ for IA. Nevertheless, it is disputable whether diagnostic advantages are sufficient against the background of a sensitivity of $0 \%$. Furthermore, an antibiotic suspension is recommended two weeks prior to aspiration in order to achieve best possible validity (33). This however might increase the risk for infection persistence and the development of drug-resistant microbial strains (34). Weighing up the diagnostic advantages and therapeutic disadvantages, we no longer perform IA in our clinic.

We acknowledge that this study has several limitations. The study design is retrospective. Including data from 2007, the definition of infection was not strictly based on 2018s ICM criteria. Due to the limited number of shoulder joint infections in general, the number of cases was limited. We included primary-, secondary- and periprosthetic infections. This heterogeneity empowers statistics but may cause a certain bias for PA, not for IA. Therefore subgroup analysis is supported as well.

\section{Conclusions}

PA aspiration is an important pillar in the diagnosis of shoulder joint infection. Whilst sensitivity is moderate, specificity is high. WBC can be low even in cases with infection. Consequently, PA cannot absolutely rule out infection. However, we strongly recommend PA for its advantages of targeted antibiotic therapy in case of germ identification as well as its major impact on establish infection scores. Surgeons should be aware that PA is likely to miss Cutibacterium spp. and CoNS. Empiric antibiotic therapy should therefore cover these bacteria even if aspiration showed negative microbiological cultures. In contrast, diagnostic value of IA seems negotiable and does not qualify for routinely use.

\section{Abbreviations}

- AUC: area under the curve

- CoNS: coagulase negative staphylococci

- IA: interstage aspiration

- ICM: International Consensus Meeting on Periprosthetic Joint Infections

- NPV: negative predictive value

- PA: preoperative aspiration

- PI: primary infection

- PMMA nolvmethvlmetacrvlate Loading [MathJax]/jax/output/CommonHTML/jax.js 
- PPV: positive predictive value

- PSI: periprosthetic shoulder infection

- ROC: receiver operating curve

- SI: secondary infection

- SJI: shoulder joint infection

- TSA: total shoulder arthroplasty

- WBC: synovial fluid white blood cell count

\section{Declarations}

Ethics approval and consent to participate

The study has been performed in accordance with the Declaration of Helsinki and has been approved by the ethic committee of the author's institution (reference number 235/16-mk). Informed consent to participate in the current retrospective study was obtained from all patients.

\section{Consent for publication}

Not applicable.

Availability of data and materials

The datasets used and/or analysed during the current study are available from the corresponding author on reasonable request.

Competing interests

The authors declare that they have no competing interests.

Funding

This publication was supported by the Open Access Publication Fund of the University of Wuerzburg.

Authors' contributions

LS: Conceptualization, Methodology, data analysis, writing. JF: Methodology, resources. SH: Review and editing, supervision. TR: Review and editing, data analysis. MR: Project administration, supervision. KR: Conceptualization, Methodology, Project administration.

All authors have read and agreed to the published version of the manuscript.

\section{$\underline{\text { Acknowledgements }}$}

Not applicable.

\section{References}

1. Bohsali KI, Wirth MA, Rockwood CA, JR. Complications of total shoulder arthroplasty. J Bone Joint Surg Am 2006, 88(10):2279-92.

2. Pulido L, Ghanem E, Joshi A, Purtill JJ, Parvizi J. Periprosthetic joint infection: the incidence, timing, and predisposing factors. Clin Orthop Relat Res 2008, 466(7):1710-5.

3. Sperling JW, Kozak TK, Hanssen AD, Cofield RH. Infection after shoulder arthroplasty. Clin Orthop Relat Res 2001, (382):206-16.

4. Zavala JA, Clark JC, Kissenberth MJ, Tolan SJ, Hawkins RJ. Management of deep infection after reverse total shoulder arthroplasty: a case series. J Shoulder Elbow Surg 2012, 21(10):1310-5.

5. Muhlhofer HML, Feihl S, Banke IJ, Suren C, Pohlig F, Eisenhart-Rothe R von. Native joint infections. Orthopade 2020, 49(2):191-200.

6. Roerdink RL, Huijbregts HJTAM, van Lieshout AWT, Dietvorst M, van der Zwaard BC. The difference between native septic arthritis and prosthetic joint infections: A review of literature. J Orthop Surg (Hong Kong) 2019, 27(2):2309499019860468.

7. Levy JC, Hasan SS. Is there a role for preoperative joint aspiration in the evaluation of a shoulder arthroplasty for periprosthetic joint infection (PJI)?: ICM Shoulder Q\&A. ICM Philadelphia Shoulder Q\&A 2018:576-7.

8. Nodzo SR, Boyle KK, Bhimani S, Duquin TR, Miller AO, Westrich GH. Propionibacterium acnes Host Inflammatory Response During Periprosthetic Infection Is Joint Specific. HSS J 2017, 13(2):159-64.

9. Strickland JP, Sperling JW, Cofield RH. The results of two-stage re-implantation for infected shoulder replacement. J Bone Joint Surg Br 2008, 90(4):4605.

10. Mook WR, Garrigues GE. Diagnosis and Management of Periprosthetic Shoulder Infections. J Bone Joint Surg Am 2014, 96(11):956-65.

11. Ince A, Seemann K, Frommelt L, Katzer A, Lohr JF. One-stage revision of shoulder arthroplasty in the case of periprosthetic infection. Z Orthop Ihre Grenzgeb 2004, 142(5):611-7. 
12. Jacquot A, Sirveaux F, Roche O, Favard L, Clavert P, Mole D. Surgical management of the infected reversed shoulder arthroplasty: a French multicenter study of reoperation in 32 patients. J Shoulder Elbow Surg 2015, 24(11):1713-22.

13. Dilisio MF, Miller LR, Warner JJP, Higgins LD. Arthroscopic tissue culture for the evaluation of periprosthetic shoulder infection. J Bone Joint Surg Am 2014, 96(23):1952-8.

14. Boelch SP, Streck LE, Plumhoff P, Konrads C, Gohlke F, Rueckl K. Infection control and outcome of staged reverse shoulder arthroplasty for the management of shoulder infections. JSES International 2020 (accepted for publication).

15. Buchalter DB, Mahure SA, Mollon B, Yu S, Kwon YW, Zuckerman JD. Two-stage revision for infected shoulder arthroplasty. J Shoulder Elbow Surg 2016.

16. Marcheggiani Muccioli GM, Huri G, Grassi A, Di Roberti Sarsina T, Carbone G, Guerra E et al. Surgical treatment of infected shoulder arthroplasty. A systematic review. Int Orthop 2017, 41(4):823-30.

17. Garrigues GE, Green, Andrew, Zmistowski, Benjamin, Hsu J, Ricchetti E, Namdari S, Frankle M et al. What are the diagnostic criteria of shoulder periprosthetic joint infection (PJI)?: ICM Shoulder Q\&A. ICM Philadelphia Shoulder Q\&A, 2018:558-65.

18. Topolski MS, Chin PYK, Sperling JW, Cofield RH. Revision shoulder arthroplasty with positive intraoperative cultures: the value of preoperative studies and intraoperative histology. J Shoulder Elbow Surg 2006, 15(4):402-6.

19. Nelson GN, Davis DE, Namdari S. Outcomes in the treatment of periprosthetic joint infection after shoulder arthroplasty: a systematic review. J Shoulder Elbow Surg 2016, 25(8):1337-45.

20. Sabesan VJ, Ho JC, Kovacevic D, lannotti JP. Two-stage reimplantation for treating prosthetic shoulder infections. Clin Orthop Relat Res 2011, 469(9):2538-43.

21. Achermann Y, Goldstein EJC, Coenye T, Shirtliff ME. Propionibacterium acnes: from commensal to opportunistic biofilm-associated implant pathogen. Clin Microbiol Rev 2014, 27(3):419-40.

22. Peterson LR. Effect of media on transport and recovery of anaerobic bacteria. Clin Infect Dis 1997, 25 Suppl 2:S134-6.

23. Pottinger P, Butler-Wu S, Neradilek MB, Merritt A, Bertelsen A, Jette JL et al. Prognostic factors for bacterial cultures positive for Propionibacterium acnes and other organisms in a large series of revision shoulder arthroplasties performed for stiffness, pain, or loosening. J Bone Joint Surg Am 2012, 94(22):2075-83.

24. Frangiamore SJ, Saleh A, Grosso MJ, Alolabi B, Bauer TW, lannotti JP et al. Early Versus Late Culture Growth of Propionibacterium acnes in Revision Shoulder Arthroplasty. J Bone Joint Surg Am 2015, 97(14):1149-58.

25. Weber P, Utzschneider S, Sadoghi P, Andress H-J, Jansson V, Muller PE. Management of the infected shoulder prosthesis: a retrospective analysis and review of the literature. Int Orthop 2011, 35(3):365-73.

26. Parvizi J, Zmistowski B, Berbari EF, Bauer TW, Springer BD, Della Valle CJ et al. New definition for periprosthetic joint infection: From the Workgroup of the Musculoskeletal Infection Society. Clin Orthop Relat Res 2011, 469(11):2992-4.

27. Dinneen A, Guyot A, Clements J, Bradley N. Synovial fluid white cell and differential count in the diagnosis or exclusion of prosthetic joint infection. Bone Joint J 2013, 95-B(4):554-7.

28. Cortes Jimenez LE. Is there a role for synovial fluid white blood cell count (WBC) and differential in the diagnosis of shoulder periprotsthetic infection (PJI)?: Shoulder Q\&A. ICM Philadelphia Shoulder Q\&A 2018.

29. Strahm C, Zdravkovic V, Egidy C, Jost B. Accuracy of Synovial Leukocyte and Polymorphonuclear Cell Count in Patients with Shoulder Prosthetic Joint Infection. J Bone Jt Infect 2018, 3(5):245-8.

30. Boelch SP, Roth M, Arnholdt J, Rudert M, Luedemann M. Synovial Fluid Aspiration Should Not Be Routinely Performed during the Two-Stage Exchange of the Knee. Biomed Res Int 2018, 2018:6720712.

31. Boelch SP, Weissenberger M, Spohn F, Rudert M, Luedemann M. Insufficient sensitivity of joint aspiration during the two-stage exchange of the hip with spacers. J Orthop Surg Res 2018, 13(1):7.

32. Holmberg A, Lood R, Morgelin M, Soderquist B, Holst E, Collin M et al. Biofilm formation by Propionibacterium acnes is a characteristic of invasive isolates. Clin Microbiol Infect 2009, 15(8):787-95.

33. Barrack RL, Jennings RW, Wolfe MW, Bertot AJ. The Coventry Award. The value of preoperative aspiration before total knee revision. Clin Orthop Relat Res 1997, (345):8-16.

34. Hangama Fayaz, Carlos A. Higuera, Igor Shubnyakov. What is the importance of two-week antibiotic holiday prior to reimplantation? International Consensus Meeting on Periprosthetic Joint Infection - Hip and Knee - Q\&A 2018:S. 383-385.

\section{Figures}




\section{Microbiological cultures from PA}

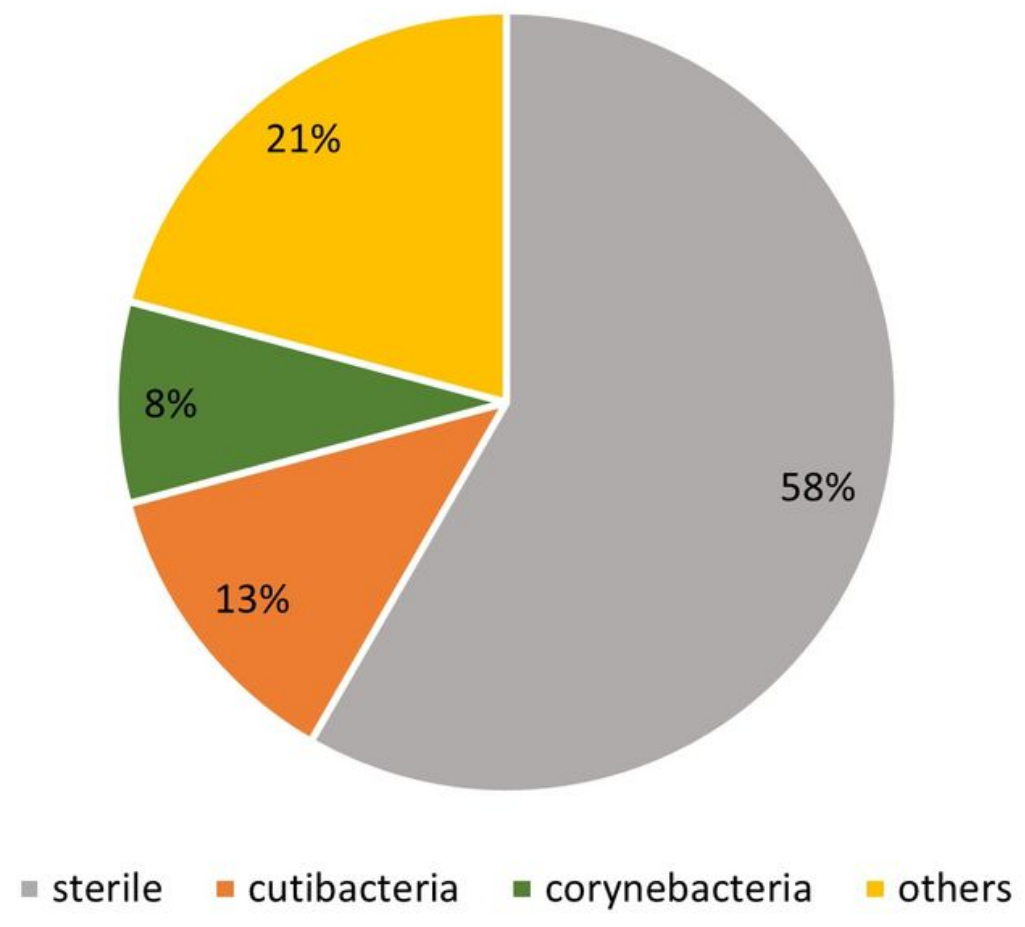

Figure 1

Results of microbiological cultures from PA (in \% of all samples). For detailed information on detected bacteria see text. 


\section{WBC}

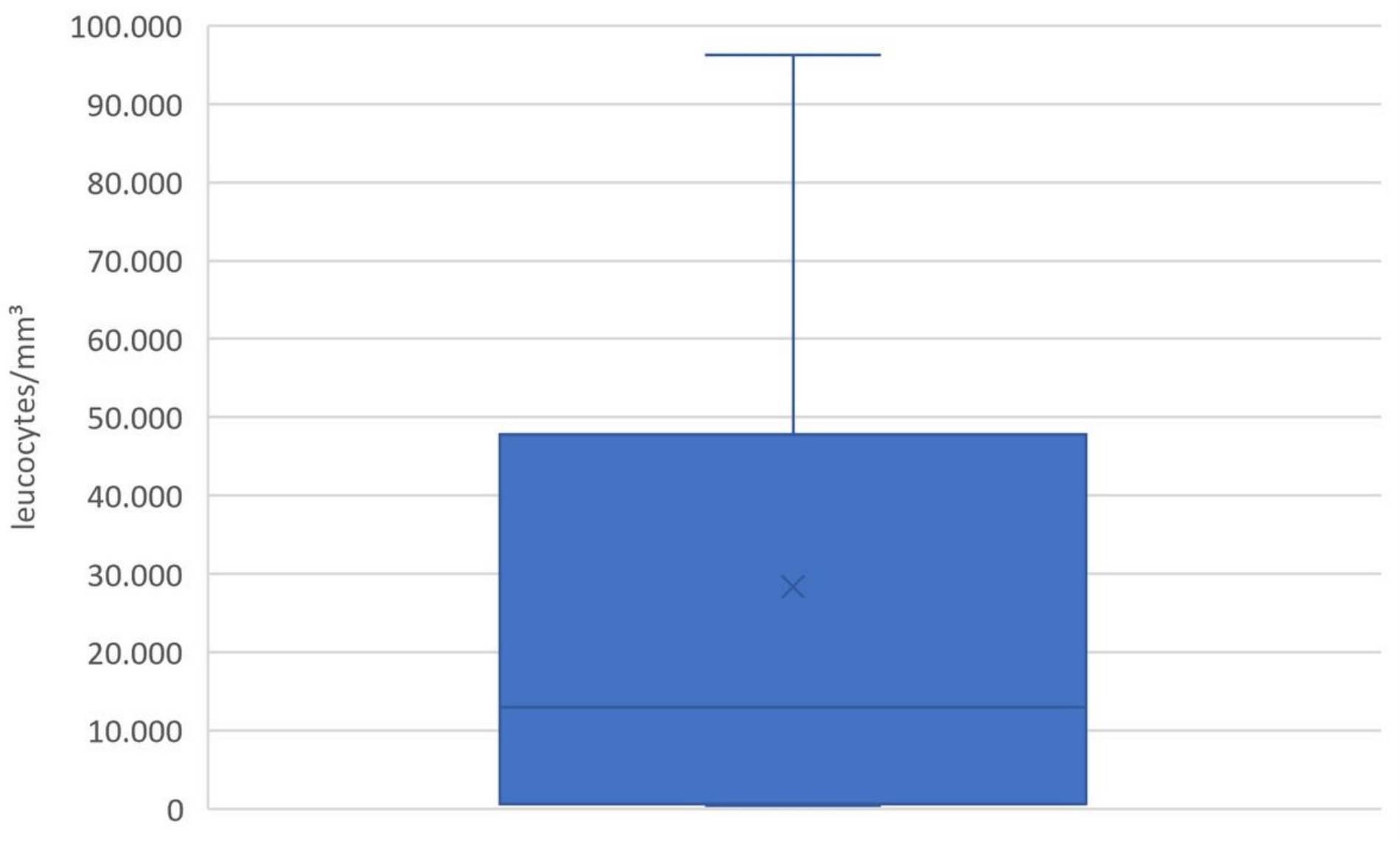

\section{Figure 2}

Synovial fluid white blood cell count in preoperative joint aspiration (WBC) in leucocytes $/ \mathrm{mm}^{3}$. Mean WBC was 27800 leucocytes/mm3 (range $400-96300$ ).

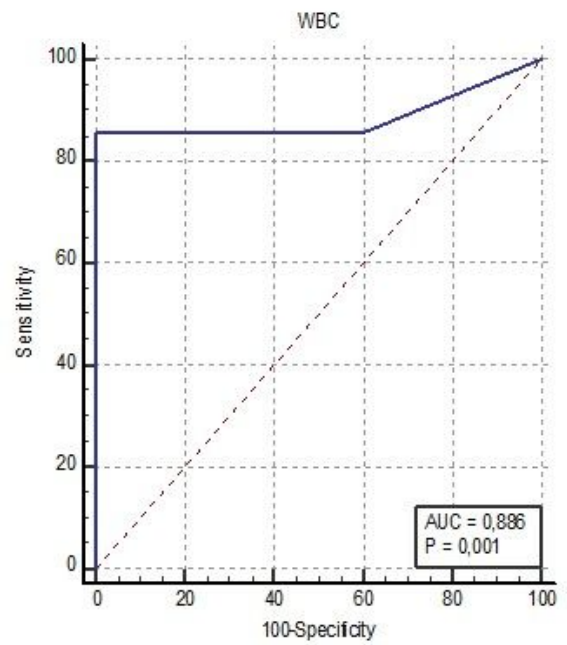

Figure 3

ROC-AUC analysis for WBC in relation to results of intraoperative microbiological culture during spacer implantation 


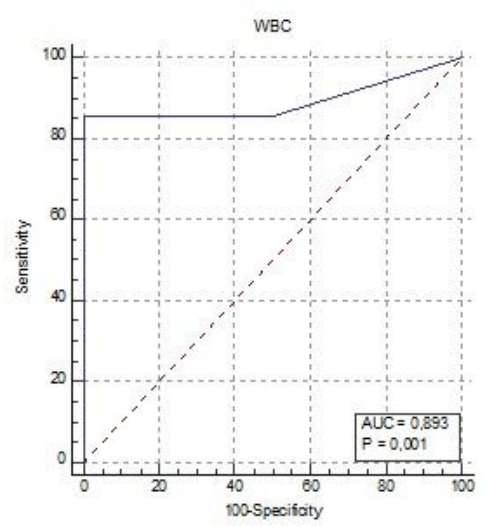

\section{Figure 4}

ROC-AUC analysis for WBC in patient with periprosthetic infection of the shoulder in relation to intraoperative microbiological culture during spacer implantation.

\section{Microbiological cultures from IA}

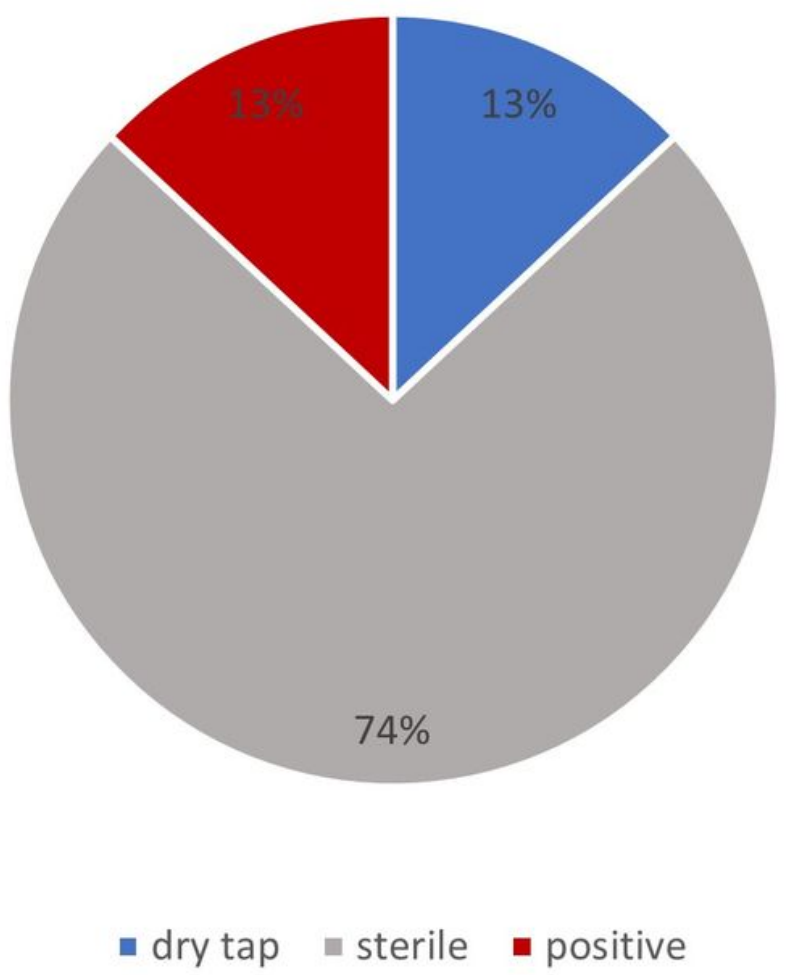

\section{Figure 5}

Results of microbiological cultures from IA (in \% of all samples). For detailed information on detected bacteria see text. 